\title{
Estrategias para promover la indagación y el razonamiento lógico en la educación primaria desde la didáctica de la Matemática
}

\author{
Strategies to Promote Research and Logical Reasoning in the Teaching of \\ Mathematics in the Schools
}

\author{
Maureen Camacho Oviedo \\ División de Educación Básica \\ Centro de Investigación y Docencia en Educación \\ Universidad Nacional \\ Heredia, Costa Rica \\ mcamac@una.ac.cr
}

Recibido 25 de julio de 2011 • Corregido 09 de enero de 2012 • Aceptado 03 de junio de 2012

\begin{abstract}
Resumen. El artículo pretende generar una reflexión sobre la imperante necesidad de que el personal docente suscite situaciones innovadoras en el proceso de enseñanza y aprendizaje en el área de la Matemática, de manera que logre promover la habilidad de indagación en el estudiantado y el desarrollo del razonamiento lógico aplicable a situaciones cotidianas. Presenta algunos datos estadísticos y posibles razones del porqué del bajo rendimiento y poco agrado hacia la asignatura de Matemática. Dado que los docentes tienen el desafío de proponer experiencias de aprendizaje significativas y funcionales para el estudiante y de generar placer por aprender, la formación docente debe incluir experiencias de este tipo para ser practicadas luego en los centros educativos de primaria. Por ello, se plantea una propuesta de trabajo desde el área de formación en la Didáctica de la Matemática que permite generar la criticidad, la curiosidad y el razonamiento lógico en el estudiantado, así como también se complementa con la temática de resolución de problemas que se debe promover y profundizar en el estudio de la asignatura de Matemática.
\end{abstract}

Palabras claves. Didáctica, razonamiento lógico, indagación, innovación, resolución de problemas, proceso de enseñanza y aprendizaje.

\footnotetext{
Máster en Educación con Énfasis en Docencia Universitaria, otorgado por la Universidad Nacional, Costa Rica. Licenciada en Ciencias de la Educación con concentración en Educación Básica I y II Ciclos. Actualmente, se desempeña como Subdirectora de la División de Educación Básica (DEB) del Centro de Investigación y Docencia en Educación (CIDE) de la Universidad Nacional (UNA), Costa Rica y como Responsable del Proyecto Desarrollo Académico y Gestión Organizacional de la División de Educación Básica. Como académica de la DEB, en la Carrera de Pedagogía con énfasis en I y II Ciclos de la Educación General Básica, se ha desempeñado en el área de docencia, en la coordinación de la Intervención Pedagógica a nivel de Diplomado, en los procesos de supervisión de la práctica a nivel de Bachillerato y como responsable de la Carrera de Pedagogía con énfasis en I y II Ciclos. Ha laborado como docente de primaria en centros educativos públicos y privados.
} 
URL: http://www.una.ac.cr/educare

\begin{abstract}
The purpose of this paper is to raise a debate on the urgent need for teachers to generate innovative situations in the teaching-learning process, in the field of Mathematics, as a way for students to develop logical reasoning and research skills applicable to everyday situations. It includes some statistical data and possible reasons for the poor performance and dissatisfaction of students towards Mathematics. Since teachers are called to offer meaningful and functional learning experiences to students, in order to promote the pleasure of learning, teacher training should include experiences that can be put into practice by teachers in the education centers. This paper includes a work proposal for Mathematics Teaching to generate discussion, curiosity and logical reasoning in students, together with the Mathematical problem solving study.
\end{abstract}

Keywords. Teaching, logical reasoning, research, innovation, mathematical problem solving, teaching and learning process.

Para quienes se involucran en el campo educativo, es comprensible que generar situaciones de innovación u optimización en el proceso de enseñanza y aprendizaje constituya un reto $y$, un reto mayor, forjar placer por aprender y fomentar la indagación en las estudiantes y los estudiantes. Estos retos se pueden intensificar según el nivel educativo y la complejidad de las asignaturas que se imparten, así como la perspectiva que tenga el estudiantado de estas.

En el entorno educativo se percibe la asignatura de Matemática como una de las más difíciles; por lo que estudiantes y padres de familia expresan que esta materia es en la que se tiene mayor temor de fracasar. Además es la asignatura que más se reprueba a nivel de primaria y secundaria. Una investigación, realizada en el 2007 por el Centro de Investigación y Docencia en Educación de la Universidad Nacional (CIDE-UNA) y planteada en el Segundo Informe del Estado de la Educación, confirma esta situación Plantea que, en relación con el proceso de transición de sexto grado a sétimo año, los alumnos entrevistados de sétimo año expresan que la materia más difícil corresponde a las Matemáticas con una cifra de 22,0\%; seguida de los Estudios Sociales, con el 17,0\%; y las Ciencias con el 12,3\%. (Programa Estado de la Nación en Desarrollo Humano Sostenible, 2008).

Además, en cuanto a los resultados de las pruebas nacionales de bachillerato, el Programa Estado de la Nación en Desarrollo Humano Sostenible (2008) menciona que, en el 2006, el porcentaje de aprobación en el ámbito nacional fue de 62,7\%, pero si se observa el rendimiento por asignatura, se exhiben porcentajes de promoción mucho más elevados que este promedio. Ejemplos de estos porcentajes corresponden a las asignaturas de Español $(96,0 \%)$ y Estudios Sociales (94,4\%). Por su parte, entre los porcentajes más bajos, se encuentran la asignatura de Matemática con un 72,2\%; Inglés, con 86,0\%; Química, con $86,1 \%$; y Física, con $86,4 \%$.

Lo anterior manifiesta que la asignatura de Matemática es una de las de más baja promoción en el estudiantado, así como refleja que, desde la perspectiva de las estudiantes y los estudiantes, la Matemática es una materia compleja. Si bien es posible investigar para determinar con más exactitud a qué se debe esta situación, se podría cuestionar si realmente al estudiantado se le hace difícil la Matemática por la naturaleza de la asignatura, por la forma en 
que se desarrolla la docencia, por el posible temor generalizado que se le tiene a esta materia y que hace que la alumna y el alumno estén predispuestos y fracasen con más regularidad o por la combinación de algunos de estos aspectos.

\section{Placer por aprender Matemática}

Se podría destacar, entonces, que se presenta otro reto que debe ser enfrentado por las educadoras y los educadores, principalmente desde la primaria, y es que, si existe un temor hacia la Matemática, es necesario modificar esta visión, sin importar si es producto de la opinión o de las experiencias del alumnado y sus padres de familia. Como docentes, debemos lograr que nuestras clases sean más atractivas y que la entrega de la docencia que se planifique sea más significativa y que, realmente, genere aprendizaje. Si se logra que los alumnos y las alumnas aprendan Matemática de una forma adecuada y placentera, tendrán más éxito en su desempeño escolar.

Un aspecto primordial para lograrlo es que el personal docente genere procesos en los cuales los escolares logren identificar la importancia y aplicación de esta asignatura en su vida diaria. Es fundamental que nuestros escolares descubran la relevancia de la Matemática en las acciones que realizan día tras día, así le darán valor como asignatura y la comprenderán más eficazmente, porque encontrarán sentido a lo que están aprendiendo y dejarán de percibir los saberes matemáticos como simples contenidos "que se dan en la escuela". Por el contrario, los percibirán como herramientas de uso diario, que les concierne manejar adecuadamente. Así no solo se manifestará como un conocimiento a nivel técnico o de aplicación, sino que el estudiantado logrará una mayor comprensión y aplicación de los conocimientos matemáticos y podrá generalizarlos al emplearlos en sus circunstancias de vida.

\section{Razones del desagrado}

Podríamos tratar de comprender algunas razones del desagrado hacia las Matemáticas y su poca comprensión. Una de ellas puede deberse a la forma de impartir los docentes las lecciones. Muchas de estas clases no poseen un adecuado proceso de construcción de las nociones matemáticas básicas y no se da un tratamiento pertinente y eficiente a sus saberes.

Debemos ser conscientes de que el centro escolar no es la única instancia para generar formación educativa, pero "(...) no puede renunciar a ser la instancia educativa que tiene la función peculiar de crear conscientemente experiencias de aprendizaje, reconocibles como tales por los sujetos implicados (...)" (Assman, 2002, p. 26). Es decir, en muchas ocasiones el ámbito escolar, y más específicamente el aula como espacio educativo, no se concibe como un 
lugar de construcción del conocimiento: el grupo de docentes no valora este espacio para crear verdaderas experiencias de aprendizajes, duraderas y significativas para los discentes. Según las experiencias observadas en las aulas y las opiniones expresadas por escolares, los conceptos se les dan ya construidos y en forma rápida. Se espera que con una breve explicación, con un ejemplo, con solo anotarlos en su cuaderno, con trabajar algún ejercicio en los libros o con una práctica, el estudiantado se apropie de ellos. Estas acciones, muchas veces, no exigen la real puesta en práctica de los conocimientos adquiridos, porque, en general, pueden ser ejercicios muy simplistas, alejados de su realidad o porque se plantean en forma compleja, sin adecuarlos a su nivel de comprensión.

Además, debemos recordar que el conocimiento del niño o niña no se establece por la imposición de un saber, sino por su participación en la construcción del conocimiento. Boule (1995) expresa que "(...) No se trata, por consiguiente, de construir lecciones alrededor de un concepto, sino de orientar la actividad o la reflexión aprovechando cada ocasión y, de vez en cuando, de manera más sintética y organizada (...)" (p. 15). Es importante, y más en el caso de esta asignatura, partir de la actividad del estudiante, de la manipulación, de la evolución y de situaciones reales y familiares, antes que imponer los conceptos desde la visión docente.

Otra posible razón para el desencanto hacia la Matemática es que a muchos maestros y maestras no les agrada impartir las clases, pues incluso para ellos es una asignatura con la que tienen poca afinidad, la comprenden limitadamente y no le encuentran la aplicación a la vida. Por ello, prefieren dar sus clases en forma monótona, repetitiva y sin profundizar en la temática, para evitar verse en desventaja ante el estudiantado o en conflictos cognitivos que no pueden manejar.

En el caso del segundo ciclo de la Educación General Básica de nuestro país (cuarto, quinto y sexto grado de primaria), las asignaturas básicas se trabajan en dos áreas: en Español y Estudios Sociales y en Matemática y Ciencias. Muchos educadores escogen tener su docencia en la primera, pues perciben la segunda como más difícil de impartir. Algunos docentes, a los que no les agrada esta asignatura, prefieren que otros se encarguen de esas lecciones, aunque por lo general esos docentes que los sustituyen en este campo puedan ser de más reciente ingreso al sistema educativo o a la institución escolar y carezcan de mayor experiencia.

En otros momentos, también en los centros educativos se tiene identificado al maestro o la maestra al que le agrada más impartir Matemática y los demás se sienten cómodos con esta situación, porque saben que ese docente manifestará más claridad y pertinencia para darles a entender a los escolares los contenidos respectivos de la asignatura y motivarles para construir juntos el conocimiento matemático, a la vez que incentiva a ese docente a seguir preparándose e innovando en el área en que se siente más seguro y que tiene mayor manejo didáctico. Sin embargo, se esperaría también que las educadoras y los educadores que perciben que la Matemática no es una de sus materias de mayor manejo en su docencia, deberían prepararse mejor, habilitándose o actualizándose más en los saberes matemáticos, para así tomar el reto de impartir, sin ningún inconveniente, esta asignatura a los estudiantes de cuarto, quinto o sexto grado de primaria. 
Lo expresado hasta este punto puede permitir entender por qué muchas veces los conceptos matemáticos son estudiados en la escuela a la ligera, sin mayor explicación o práctica, sin intención de profundizar o aplicar, lo que genera que las clases de Matemática sean monótonas, indiferentes y sin intencionalidad. Por consiguiente, no se hace prioritario promover la curiosidad ni la iniciativa en los estudiantes, quienes continuarán en sus clases en espera de resolver los ejercicios y problemas matemáticos con procedimientos que les sean dados y no con métodos de resolución que ellos mismos puedan generar o construir con sus compañeros, compañeras y docente.

Cabe indicar que muchas veces se pretende señalar al estudiante como el único responsable de su rendimiento en las diversas asignaturas, en este caso en Matemáticas por ser una materia más práctica que memorística; por supuesto, el escolar debe ir construyendo sus propias nociones, pero a partir de las herramientas y orientaciones proporcionadas por el docente. Es decir, se debe construir en conjunto, valorando el maestro o la maestra que las vivencias del niño o la niña son constantes y complejas, llenas de descubrimientos e interrogantes; aceptando que si el docente no saber qué enseñar o cómo hacerlo, limita el ritmo de las experiencias de aprendizaje del escolar, lo desubica, desorganiza sus acciones y el estudiantado no alcanzará el saber matemático fundamental.

\section{Matemática y formación docente}

Una manera de impulsar el agrado por la Matemática y una mediación didáctica más pertinente es fomentarlo en los docentes y las docentes de primaria que están en la formación universitaria, quienes serán los responsables de trasmitir ese gusto hacia la asignatura a sus escolares y el reconocimiento sobre la importancia que tiene esta en la vida cotidiana. Para ello, es importante considerar ciertas premisas que permitan permear la formación docente en todas las áreas del saber y no solo en el conocimiento matemático. Algunas implicaciones didácticas a considerar aportadas por Bejarano et al. (2004) son las siguientes:

- Relación teórico práctica

- Didáctica reflexiva

- Didáctica contextualizada

- Didáctica participativa

- Didáctica comunicativa

La relación teórico práctica permite, según las autoras, que surja el concepto de praxis, como el término que posibilita unificar la relación teoría-práctica y ese vínculo se produce en la interacción entre el contexto y los participantes del proceso educativo. En la fundamentación de los Planes de Estudio de la División de Educación Básica (DEB) del Centro de Investigación 
URL: http://www.una.ac.cr/educare

y Docencia en Educación (CIDE) de la Universidad Nacional, se plasma el concepto de praxis como "(...) un proceso de acción y de reflexión, en diálogo permanente con la realidad y con los fundamentos que permiten su lectura, desarrollo y su verdadero significado en el proceso mismo de intervención y con la participación de todos los actores involucrados" (UNA, Centro de Investigación y Docencia en Educación. División de Educación Básica, 2005, p. 26).

La didáctica reflexiva plantea que el proceso de reflexión es el que nos permite tomar conciencia de nuestra práctica pedagógica y darle significado. Por ello, es importante "(...) revisar y repensar lo que hemos hecho en nuestras prácticas de enseñanza, apoyados en marcos teóricos que nos ayuden a encontrar el sentido de dichas prácticas y comprender la realidad" (Bejarano et al., 2004, p. 65). En el caso de la asignatura de Matemática, es importante razonar no solo respecto del manejo teórico-práctico de los saberes matemáticos propios del nivel de primaria de los docentes, sino también sobre la metodología y estrategias de aprendizaje que se están generando en nuestros espacios educativos.

La didáctica contextualizada le asigna a la didáctica un carácter social y singulariza las situaciones educativas, pues cada clase, cada estudiante, cada ambiente educativo es único y particular. Lo importante es "(...) atender las circunstancias que cada caso presenta y no pretender la uniformidad de los procesos educativos de los niños y las niñas" (Bejarano et al., 2004, p. 66). Esta implicación didáctica le permite al docente, junto con sus alumnos y alumnas, crear espacios únicos, irrepetibles, pero significativos, en los que se generalice lo aprendido a otros ámbitos más allá del aula. Por ello, es importante que el educador fomente, en sus clases, la curiosidad, la experimentación, la resolución de problemas, respetando las particularidades de sus estudiantes y del contexto, y aprovechando este último como insumo y medio para poner en práctica lo aprendido y generado en clase.

La didáctica participativa señala que el proceso de enseñanza y aprendizaje es un proceso social e interactivo, que debe propiciar la reflexión y el análisis entre los actores sociales (Bejarano et al., 2004). Esto le permite al docente en formación y al docente en ejercicio comprender que el niño no aprende solo, aprende con otros, al compartir con los demás sus conocimientos y sus dudas, al construir conocimientos con sus iguales y con sus docentes. El escolar reflexiona sobre lo que aprende y cómo lo aprende.

La didáctica comunicativa indica que las prácticas de enseñanza deben ser objeto de discusión y reflexión entre los docentes. Bejarano et al. (2004) señala que un "(...) docente que reflexiona su práctica, la analiza, puede estar en mayor capacidad de mejorar sus cualidades pedagógicas, de renovar e innovar, de tomar decisiones, de proponer, de ser un docente que transforme y mejore su quehacer pedagógico" (p. 66). Esto significa que en la formación docente universitaria se deben abordar los procesos de enseñanza y aprendizaje de una forma diferente, en la que se experimenten procesos didácticos significativos, para que el educador en formación se apropie de ellos y los genere posteriormente con sus discentes, manteniendo una constante reflexión sobre ellos y discutiendo los mismos con el grupo de alumnos y el grupo de profesores, con el fin de enriquecer estas experiencias. 


\section{Propuesta de curiosidades matemáticas dentro de los cursos de formación docente}

En las líneas siguientes se comenta una experiencia respecto de la mediación didáctica, referida a una de las propuestas llevadas a cabo en el curso universitario Didáctica de la Matemática para la Educación Básica (Camacho, 2010), ofrecido por la División de Educación Básica de la Universidad Nacional. Dentro de las temáticas del curso está el promover principios didácticos en el abordaje de la Matemática, incluyendo el gusto por la asignatura al identificar su importancia y funcionalidad en el ámbito cotidiano, respetando el contexto en el que se enmarca y promoviendo la generalización de los aprendizajes. Esto incluye la promoción de espacios educativos significativos y lúdicos, en los que se apliquen diferentes recursos de enseñanza y aprendizaje, todo como parte de la formación de los futuros educadores y educadoras. Este curso se ubica en el segundo año del Nivel de Diplomado de la carrera en Pedagogía en I y II Ciclos de la Educación General Básica.

Como parte de la organización del curso, en las diferentes sesiones de clase y según previa distribución, los estudiantes-docentes presentan al resto del grupo algún o algunos ejercicios, en los que deban poner a prueba la lógica matemática, la curiosidad y la imaginación. Para ello, la profesora o profesor debe impartir una clase modelo en las lecciones iniciales y luego dar la oportunidad a los estudiantes-docentes para que investiguen en diversas fuentes algún tipo de ejercicio o de situación-problema curiosa, el cual genere razonamiento y uso de la lógica. Cada estudiante aporta esta situación-problema para ser compartida y resuelta por sus compañeros de clase, en un lapso de quince minutos aproximadamente. La idea pretende que estos ejemplos, presentados en el aula universitaria, a la vez sirvan para ser aplicados a nivel de primaria y relacionados, por el propio escolar o el docente, con ciertos contenidos matemáticos de los diversos grados.

Lo anterior permite que el estudiantado universitario vaya conformando un banco de recursos matemáticos que le servirán para implementar en las escuelas, relacionados siempre con los temas que deben estudiarse, según el Programa de Estudio de Matemática del Ministerio de Educación Pública. Un aspecto relevante de estas situaciones-problemas curiosas es que pueden aplicarse en diferentes momentos del proceso de enseñanza y aprendizaje de la Matemática; pueden servirle al educador o educadora para motivar al inicio del estudio de un tema, así como durante el desarrollo de la temática o bien como cierre de la misma; en cualquiera de los momentos, es un tipo de práctica matemática presentada de una forma diferente a la usual. Estos ejercicios ${ }^{2}$ permiten al docente evaluar la comprensión del problema, así como analizar el nivel de lógica de los niños y las niñas, su nivel de independencia al resolver problemas; aspectos que desde la didáctica reflexiva y participativa pueden ser analizados por el docente o la docente y su grupo de alumnos y alumnas; a la vez, al ser una instrucción tipo problema diferente a los tradicionales, constituye un entretenimiento para el estudiantado y promueve su imaginación y curiosidad natural.

Para este artículo se utiliza la palabra ejercicio y situación problema como similares, en el sentido de que se pretende que los escolares resuelvan ejercicios que les requieran un razonamiento y búsqueda de soluciones, mediante el uso de la lógica, es decir, ejercicios tipo problema, pero sin enmarcarlos en el concepto tradicional de problema como aquel que se resuelve únicamente con operaciones fundamentales y plasmando el planteo, la operación y la respuesta; sino como pequeños problemas curiosos que representen un reto intelectual. 
URL: http://www.una.ac.cr/educare

Asimismo, los estudiantes y las estudiantes universitarias, al presentar su propuesta, deben explicar la forma de resolución. Esto se hace primero compartiendo las ideas de resolución logradas por el grupo universitario y, luego, en forma más dirigida por el estudiante-docente encargado o encargada del ejercicio o situación-problema. Además, debe sugerir cómo aplicar la actividad-problema en la escuela, para cuáles temáticas escolares se puede ajustar y para cuáles grados lo considera pertinente. A este tipo de estrategia didáctica del curso se le denomina curiosidades matemáticas, ya que se pretende fomentar la curiosidad e interés hacia ejercicios que promueven la iniciativa, el razonamiento y la lógica matemática.

Para trabajar este tipo de curiosidad matemática, se debe ser constante en su utilización en el aula escolar, pues si los niños y niñas no han estado en contacto con este tipo de instrucción antes, pueden sentirse presionados y con dificultad para resolverlo. De allí que también es necesario iniciar con situaciones problema-sencillas, que no requieran de muchos pasos para su solución. Esto, por cuanto otro objetivo de esta estrategia es promover la lógica con planteamientos de resolución de problemas diferentes del tradicional -por lo general solo con el uso de las operaciones fundamentales básicas-. Se espera que el escolar perciba la necesidad de probar con otras formas de resolución, usar el tanteo, la probabilidad, la prueba y error y compartir sus propuestas e ideas con su grupo de iguales. Esta actitud generará la idea de que cada quien es responsable en la construcción de su aprendizaje, así como partícipe del aprendizaje de sus compañeros y compañeras, con la guía y sapiencia del docente.

Algunas curiosidades matemáticas, por ejemplo, que permiten desarrollar el razonamiento lógico, pueden estar relacionados con:

- Interpretación de jeroglíficos o dibujos

- Ordenamiento de cifras para obtener un resultado definido

- Discriminación entre figuras o complete de series de figuras

- Acertijos sobre frases con números o colocación de números en cierta posición para obtener cantidades establecidas

- Juegos de palabras y números para obtener fracciones o múltiplos de ciertas cantidades

- Identificación de enunciados falsos, pues matemáticamente son incorrectos

- Ordenamiento de pasos o colocación de objetos y líneas en ciertas posiciones, entre otros. 


\section{Fomento de la resolución de problemas}

En el curso de Didáctica de la Matemática para la Educación Básica también se recalca la importancia de fomentar en la escuela la resolución de problemas de la forma más pertinente posible, pues muchas veces se limita al estudiante en la toma de decisiones y al darles la forma de resolución, lo cual no favorece el intento de buscarla. Se puede considerar que en los escolares no se ejercita lo suficiente esta habilidad para la comprensión y resolución de problemas, situación que les genera dificultades a futuro dentro del sistema educativo e incluso en la vida diaria. Por ello, como parte del curso universitario, se complementa también el tema de la resolución de problemas con indagación teórica sobre la temática y entre la información que se proporciona se encuentra la teoría de Pólya (Pólya, citado por Alfaro (2006). Además, la fundamentación teórica que se trabaja en las sesiones de clase se relaciona con la relevancia de poner en práctica las curiosidades matemáticas, para contrastar esa visión estructurada o "cuadrada" de lo que es un problema y presentarlo a los estudiantes como una situación problemática que requiere una completa estrategia de resolución.

También, considerando que cada día y en diversos contextos cotidianos se enfrenta este tipo de situaciones que requieren de nuestra lógica, conocimiento e ingenio para su solución, se puede partir de una situación sencilla hasta una más compleja. Por ejemplo, desde organizar una ruta para salir de la casa y llegar a un determinado destino, hasta estructurar todo un presupuesto familiar atendiendo los requerimientos de todos sus miembros y en concordancia con la situación económica de la familia y del país.

Pólya, citado por Alfaro (2006), expresa que su punto de vista es que “(...) la parte más importante de la forma de pensar que se desarrolla en matemática es la correcta actitud de la manera de cometer y tratar los problemas, tenemos problemas en la vida diaria, en las ciencias, en la política, tenemos problemas por doquier (...)" (p. 28). Para Pólya lo principal en la enseñanza de la Matemática es desarrollar tácticas en la resolución de problemas, pues nos enfrentamos a los problemas en todo momento, van a requerir de nuestro razonamiento e intuición para resolverlos y los escolares deben analizar estas situaciones-problema como ejercicios que permiten construir su aprendizaje y no solo como simples contenidos que deben aprender para una prueba sumativa.

\section{Pasos lógicos para la resolución de problemas}

En cuanto a los pasos para resolver un problema, se pueden indagar propuestas de diferentes autores o se pueden construir con los escolares, según sus conocimientos previos y experiencias educativas. El docente, quien es el que conoce las particularidades del grupo, es el que puede definir cuál estrategia utilizar y cómo ejecutarla con el estudiantado. Al respecto, un ejemplo de teoría para la resolución de problemas es la que plantea George Pólya, como una sucesión de pasos lógicos para aplicar en la resolución de cualquier tipo de problema. Diseña, en su método, cuatro pasos o etapas y 
para cada una de esas etapas plantea una serie de preguntas y sugerencias. Del método de los cuatro pasos de Pólya, citado por Alfaro (2006, pp. 28-31) podemos interpretar los pasos principales a seguir y las posibles interrogantes que se pueden realizar para ser aplicadas por el propio estudiantado o por este con el apoyo docente. Dichos pasos son los siguientes:

\section{Comprender el problema (...)}

- ¿Cuál es la incógnita?

- ¿Cuáles son los datos?

- ¿Cuál es la condición?

- ¿Es la condición suficiente para determinar la incógnita?

- ¿Es insuficiente?

- ¿Es redundante?

- ¿Es contradictoria? (...)

\section{Concebir un plan (...)}

- ¿Se ha encontrado con un problema semejante?

- ¿Ha visto el mismo problema planteado en forma ligeramente diferente?

- ¿Conoce un problema relacionado?

- ¿Conoce algún teorema que le pueda ser útil?

- ¿Podría enunciar el problema en otra forma?

- ¿Podría plantearlo en forma diferente nuevamente? (...)

\section{Ejecución del plan (...)}

- ¿Puede ver claramente que el paso es correcto?

- ¿Puede demostrarlo? (...)

\section{Examinar la solución}

- ¿Puede verificar el resultado?

- ¿Puede verificar el razonamiento?

- ¿Puede obtener el resultado en forma diferente?

- ¿Puede verlo de golpe?

- ¿Puede emplear el resultado o el método en algún otro problema? (...). (pp. 28-31) 
Se logra interpretar que el primer paso sugiere establecer cuál es la incógnita del problema, los datos y las condiciones con las que se cuentan. Posterior a esto, se debe concebir el plan, relacionándolo con otras situaciones o resultados similares que puedan ser útiles en la resolución del problema. Este paso puede ser de dificultad para los estudiantes, máxime si la resolución de problemas no es una estrategia permanente facilitada por el docente, así que se sugiere que al inicio sea una interpretación grupal del problema planteado, para que el estudiante vaya familiarizándose con la estrategia y posteriormente logre interpretar y construir diversas formas de resolución de problemas.

En la etapa de ejecución del plan es muy importante revisar todos los detalles e identificar la diferencia entre percibir que un paso es correcto y demostrar que lo es. De allí la importancia de interpretar adecuadamente la incógnita; utilizar la intuición, la experimentación, el razonamiento lógico; ejecutar las estrategias de solución y compartirlas con la clase.

En la última etapa, también denominada como la etapa de la visión retrospectiva, es muy importante revisar lo que se realizó, verificar el resultado y el razonamiento seguido, pues esto permitirá no solo resolver un problema, sino que puede generar habilidades para resolver posteriores situaciones, o sea, se podría utilizar el método de solución como medio para hacerle frente a otro tipo de problemas. Además, al examinar y compartir la solución se logra verificar que puede existir otras formas de obtener los resultados, lo que recalcaría que existen varias posibilidades de resolver un mismo problema, hecho indispensable de enseñar al estudiantado. La riqueza en las estrategias de solución permitirá generar más entendimiento y creatividad en los estudiantes, al compartir su análisis se fomentarán más conocimientos, razonamiento lógico y generalizaciones en los alumnos y alumnas.

Al seguir esta estrategia de los cuatro pasos de Pólya, se puede incentivar en los estudiantes un mayor gusto por la resolución de problemas y una mayor comprensión de la importancia de dicha habilidad para situaciones de la vida diaria. Esta estrategia podría utilizarse, si se considerase necesario, en la resolución de las curiosidades matemáticas, pues representan situaciones -problemas de diverso nivel y requieren una solución meditada y planificada, con su correspondiente verificación, aunque se debe considerar que estas, además, incluyen la parte de curiosidad y experimentación.

Es importante recomendar, además, que si el docente decide utilizar los cuatro pasos de Pólya en su clase, él o ella debe ser el responsable de aclarar y contextualizar la terminología a los escolares, los pasos e incógnitas planteadas son un referente para el educador o educadora, pero como parte de su rol como mediador del aprendizaje debe orientar a los estudiantes y a las estudiantes, y situar la teoría según el nivel escolar. Debe orientar a sus alumnos y alumnas, en primera instancia, ejecutando él o ella la teoría y luego facilitar para que sus alumnos y alumnas puedan realizar, por su parte, los pasos propuestos por Pólya, así como también dejar espacios para solucionar los problemas en forma conjunta. Todas estas sugerencias quedan a criterio del educador o educadora, quien es quien conoce más cercanamente a su grupo de estudiantes, su nivel y su ritmo de aprendizaje y de construcción de conocimiento. 
URL: http://www.una.ac.cr/educare

\section{Consideraciones finales}

Es importante, como docentes, generar estrategias diversas para lograr un enlace entre los saberes conceptuales y procedimentales de la asignatura de Matemática con la realidad e intereses de los escolares. Se debe lograr un adecuado andamiaje entre los conocimientos previos que poseen los estudiantes y los conocimientos nuevos por promover en cada nivel escolar, de manera que les sean más significativos y funcionales en su vida cotidiana. Se lograría, así, un mayor reconocimiento del rol de la Matemática en el quehacer diario de las personas, así como un disfrute en el proceso de enseñanza y aprendizaje de dicha asignatura.

El fomento del área lógico matemática plantea el reto de generar estrategias encaminadas a despertar el interés subyacente en el niño o niña, así como la construcción por parte del escolar de sus propios conceptos lógico matemáticos, que le permitirán ser una persona intelectualmente autónoma, con sus propias ideas y sus propias propuestas, respetuosa de las ideas de las otras personas, capaz de situarse en otros puntos de vista y de dar supuestos ante diversas situaciones; dueña y constructora de su propio pensar.

El rol docente debe ser mediador del aprendizaje, generador de un ambiente intelectual propicio para el estudiantado. Para contribuir con ello, se puede contar con variedad de situaciones-problema utilizadas como curiosidades matemáticas que estimulen al estudiante a hacer sus propias construcciones. Se reitera que pueden ser utilizadas en la formación universitaria de los futuros docentes, así como en los diversos grados a nivel escolar. Dichos ejercicios pueden encontrarse en fuentes impresas o digitales. Lo importante es recurrir a múltiples recursos, para ello se necesita indagar y planificar la utilización de las curiosidades o situaciones problema identificando claramente los propósitos didácticos que se alcanzarían o desarrollarían con ese tipo de ejercicios, así como el disfrute y promoción de la creatividad y la curiosidad que se podría generar en los estudiantes.

La ejecución de la propuesta de curiosidades matemáticas podrá permitir el desarrollo del razonamiento lógico, la indagación, así como la oportunidad de generar una visión diferente de la Matemática por parte del estudiantado y sus docentes, de manera que se capte su importancia desde otra mirada. 


\section{Referencias}

Alfaro, C. (noviembre, 2006). Las ideas de Pólya en la resolución de problemas. Cuadernos de Investigación y Formación en Educación Matemática, 1, 27-45. Recuperado de http://imm. ucr.ac.cr/ojs/index.php/CIFEM/article/view/3/6

Assman, H. (2002). Placer y ternura en la educación. Hacia una sociedad aprendiente. Madrid, España: Narcea ediciones.

Bejarano, S., Castro, M., Hernández, A. M., Herrera, A., Oviedo, G., Ruiz, S. y Vargas, R. (2004). Reflexiones en torno a la didáctica: Implicaciones en la formación docente. Revista Educare, 6, 59-67.

Boule, F. (1995). Manipular, organizar, representar. Iniciación a las Matemáticas. Madrid: Nárcea de Ediciones.

Camacho, M. (2010). Didáctica de la Matemática para la Educación Básica (DBJ 209). División de Educación Básica, Universidad Nacional.

Programa Estado de la Nación en Desarrollo Humano Sostenible. (2008). Estado de la Educación 2. San José, Costa Rica: CONARE.

Universidad Nacional, Centro de Investigación y Docencia en Educación. División de Educación Básica. (2005). Plan de estudios en Pedagogía con énfasis en I y ll ciclos de la Educación General Básica. Heredia: Autor. 


\section{Apéndice}

\section{Ejemplo de resolución de problema según los cuatro pasos de Pólya}

\section{Enunciado del problema:}

Un comerciante desea realizar una pequeña remodelación en su negocio para instalar más estantes, colocar más productos y atraer más clientes. Para ello, reorganiza sus gastos y decide ahorrar el $10 \%$ de la ganancia obtenida en los productos comestibles. En un mes el comerciante obtuvo una ganancia de 154320 colones en productos de limpieza, de 332500 colones en víveres y 83450 colones en productos de librería. ¿Cuánto dinero tendrá que ahorrar aún el comerciante, si se requiere para la remodelación un total de 225000 colones? $^{3}$

\section{Analicemos los pasos:}

\section{Comprender el problema}

¿Cuál es la incógnita?

$\checkmark$ La cantidad de dinero que le falta por ahorrar al comerciante.

¿Cuáles son los datos?

$\checkmark$ Las cifras o cantidades que se plantean en el enunciado del problema. Se pueden identificar todos los datos presentes en el problema, pero luego debe discriminarse cuáles de ellos son útiles para resolverlo.

$\checkmark$ Datos:

a) El porcentaje de ahorro: $10 \%$.

b) Las ganancias obtenidas:

154320 colones en productos de limpieza.

332500 colones en víveres.

83450 colones en productos de librería.

c) Costo total de la remodelación: 225000 colones.

¿Cuál es la condición?

$\checkmark$ Se requiere averiguar el porcentaje de ahorro, pues dicha cantidad es con la que el comerciante podrá disponer para hacer la remodelación.

\footnotetext{
El enunciado del problema fue elaborado por la autora del presente artículo.
} 
¿Es la condición suficiente para determinar la incógnita? ¿Es insuficiente? ¿Es redundante? ¿Es contradictoria?

$\checkmark$ Con los datos que se tienen y siguiendo un procedimiento adecuado, es suficiente la condición para determinar la incógnita.

\section{Concebir un plan}

¿Se ha encontrado con un problema semejante?

$\checkmark$ Esta pregunta depende de los conocimientos previos del estudiantado y del grado escolar en el que se encuentre. Se podría suponer que si se ubican en un quinto o sexto grado de primaria, pudieron ya haber visto o realizado problemas semejantes.

¿Ha visto el mismo problema planteado en forma ligeramente diferente?

$\checkmark$ Es probable que estudiantes de II Ciclo de la Educación General Básica (EGB) costarricense hayan visto problemas semejantes, en cuanto a tener que averiguar datos que faltan, o datos iniciales que deben determinarse para poder resolver las siguientes partes del problema, 0 problemas que requieren de la realización de varias operaciones fundamentales.

¿Conoce un problema relacionado?

$\checkmark$ Es probable que los estudiantes expresen situaciones que han visto en la vida diaria, como por ejemplo, visita a una tienda con sus padres donde vendían productos con porcentajes de descuento, o que en su familia dedican una parte del dinero al ahorro, sea sus padres, sus hermanos o el mismo estudiante.

Tanto esta interrogante como las dos anteriores, pueden ser mediadas por el docente o la docente, para identificar los conocimientos previos con los que cuenta el estudiantado, al respecto solo se anotan aquí posibles respuestas al ser una propuesta o ejemplo.

¿Conoce algún teorema que le pueda ser útil?

$\checkmark$ En este punto se puede verificar si los estudiantes y las estudiantes conocen sobre el procedimiento para obtener porcentajes y realizar algoritmos de las operaciones fundamentales. Si el profesorado con las respuestas de los escolares y las escolares considera que se debe reforzar los teoremas, algoritmos o fórmulas, en este punto se propone que lo realice.

¿Podría enunciar el problema en otra forma?

$\checkmark$ Un ejemplo podría ser el siguiente: Un vendedor quiere remodelar su negocio, por eso va a ahorrar el 10\% de lo que gane con la venta de productos comestibles o víveres. Si en un mes obtuvo una ganancia de 332500 colones en víveres. ¿Cuánto dinero le falta por ahorrar, si en total ocupa para la remodelación 225000 colones? 
URL: http://www.una.ac.cr/educare

¿Podría plantearlo en forma diferente nuevamente?

$\checkmark$ Un ejemplo podría ser el siguiente: Un vendedor quiere remodelar su negocio, por eso va a ahorrar la décima parte de las ganancias que va a obtener con la venta de comestibles o víveres. Si en un mes obtuvo una ganancia de 332500 colones en víveres. ¿Cuánto dinero le falta aún por ahorrar, si en total ocupa para la remodelación 225000 colones?

\section{Ejecución del plan}

¿Puede ver claramente que el paso es correcto?

$\checkmark$ Las dos últimas preguntas del paso 2 le permitirán al estudiantado estar más claro sobre qué es lo que debe averiguar y cómo hacerlo. Además, le permiten al docente identificar si los escolares y las escolares están comprendiendo de qué trata el problema y si comprenden qué deben hacer. Teniendo esto claro, podrán definir los procedimientos a seguir de forma correcta.

¿Puede demostrarlo?

$\checkmark$ En este punto el estudiantado podrá verbalizar o escribir lo que crea que deba hacer y lo demostrará ejecutando los procedimientos que considere necesarios. Esta demostración se refiere a una categoría de aplicación, que los escolares y las escolares de un nivel de II Ciclo de la EGB podrían realizar. Es aplicar las estrategias que consideren que les permitirán resolver el problema.

$\checkmark$ En el caso de este problema los procedimientos incluyen:

a) Obtener el 10\% de 332 500, para ello aplicarían la fórmula para obtener porcentajes: 332500 X 10 / 100, lo que dará como resultado 33250 colones.

No se debe sacar el 10\% de las otras cantidades (154 320 colones ni 83450 colones), pues se refieren a otro tipo de productos que no son de la categoría de comestibles.

b) Luego, comprendiendo que el total para la remodelación es 225000 colones y que el comerciante en ese mes logró ahorrar 33250 colones, se debe realizar una resta: 225000 - 33 250, lo que dará como resultado 191750 colones.

c) Seguidamente, es recomendable leer de nuevo la pregunta y contestarla según los resultados obtenidos.

\section{Examinar la solución}

¿Puede verificar el resultado?

$\checkmark$ Es muy importante la verificación, en especial para que el estudiantado se sienta seguro de los resultados que obtuvo. En este caso, debe verificarse lo del porcentaje y la resta. 
Para ello, por ejemplo, puede multiplicarse $33250 \times 10$, pues el $10 \%$ es lo mismo que obtener la décima parte de una cantidad, además de comprender que la multiplicación es la operación inversa de la división.

En el caso de la resta, por ejemplo, comprendiendo que la suma es su operación inversa, podemos sumar 191750 con 33250 para verificar que el resultado sea 225000 . Si no lo es, se entiende que hubo un error en el desarrollo de la resta y se debe proceder a realizarla de nuevo.

¿Puede verificar el razonamiento?

$\checkmark$ Se puede revisar el razonamiento realizado, sea individualmente, en parejas, en grupo o con la mediación del profesorado. Sea el resultado obtenido el correcto o no, esta revisión es muy importante.

$\checkmark$ Por ejemplo, en el caso de este problema, se debe verificar, por parte de los escolares o las escolares, o con el apoyo del profesorado, que no se hayan utilizado los otros datos del problema, pues no se requerían. Pueden considerarse como distractores o aspectos que amplían el enunciado del problema para contextualizarlo mejor.

¿Puede obtener el resultado en forma diferente?

$\checkmark$ Es importante que los escolares y las escolares comprendan que, por lo general, hay varias formas de resolver un problema, influyen los conocimientos propios del individuo, su formación escolar, las experiencias de mediación pedagógica que haya tenido la oportunidad de tener durante el proceso. Esto mismo debe tenerlo claro el profesor o profesora, pues puede ser que como experto tenga una idea preconcebida de cómo resolver un problema en particular, pero los estudiantes y las estudiantes pueden visualizar y elaborar otras formas también correctas de llegar al resultado esperado.

$\checkmark$ Por ejemplo para este problema, puede decidirse dividir la cantidad de 332500 entre 10, obteniendo 33250 colones, y por cálculo mental hacer la diferencia entre 225000 y 33 250, obteniendo el mismo resultado.

¿Puede verlo de golpe?

$\checkmark$ Esto dependerá de cada individuo, es probable que para parte del estudiantado sea sumamente claro identificar qué es lo que debe hacer, pero otros requerirán más tiempo y análisis del enunciado.

¿Puede emplear el resultado o el método en algún otro problema?

$\checkmark$ Es muy posible que recuerde este procedimiento en problemas referidos a porcentajes, a cálculo de diferencias entre cantidades. 